This item was submitted to Loughborough's Research Repository by the author.

Items in Figshare are protected by copyright, with all rights reserved, unless otherwise indicated.

\title{
Tailoring strongly nonlinear negative stiffness
}

\section{PLEASE CITE THE PUBLISHED VERSION}

http://dx.doi.org/10.1115/1.4025794

\section{PUBLISHER}

(C) AMSE

\section{VERSION}

AM (Accepted Manuscript)

\section{PUBLISHER STATEMENT}

This work is made available according to the conditions of the Creative Commons Attribution-NonCommercialNoDerivatives 4.0 International (CC BY-NC-ND 4.0) licence. Full details of this licence are available at: https://creativecommons.org/licenses/by-nc-nd/4.0/

\section{LICENCE}

CC BY-NC-ND 4.0

\section{REPOSITORY RECORD}

Liu, F.H., Stephanos Theodossiades, D.M. McFarland, A.F. Vakakis, and L.A. Bergman. 2019. "Tailoring Strongly Nonlinear Negative Stiffness". figshare. https://hdl.handle.net/2134/22516. 


\title{
Tailoring Strongly Nonlinear Negative Stiffness
}

\section{F. Liu' ${ }^{1}$, S. Theodossiades ${ }^{2}$, D.M. McFarland ${ }^{3}$, A.F. Vakakis ${ }^{4}$ and L.A. Bergman ${ }^{3}$}

1. Department of Automotive Engineering, Yancheng Institute of Technology, Yancheng, 224051, P.R. China, email: fuhaoliu@mvrlab.com

2. Department of Mechanical Engineering, Loughborough University, Loughborough, LE11 3TU, UK, email: S.Theodossiades@lboro.ac.uk

3. Department of Aerospace Engineering, University of Illinois at Urbana-Champaign, Urbana, IL 61801, USA, email: dmmcf@illinois.edu \& Ibergman@illinois.edu

4. Department of Mechanical Science and Engineering, University of Illinois at Urbana-Champaign, Urbana, IL 61801, USA, email: avakakis@illinois.edu

\begin{abstract}
Negative, nonlinear stiffness elements have been recently designed as configurations of pairs or groups of linear springs. We propose a new design of such a system by using a single linear spring with its moving end rolling on a path described by an equation of varying complexity. We examine the effect that the selection of the path has on the size of the deflection regime where negative stiffness is achieved. The stability properties of the equilibrium positions of the system are also investigated, highlighting the influence that the complexity of the path equation brings. The latter naturally affects the characteristics of the forcing functions around these positions. It is demonstrated that the properties of the system can be tailored according to the nature of the equation used and we show how essentially nonlinear negative stiffness elements, (i.e., with no linear parts) can be designed. These results provide a useful standpoint for designers of such systems, who wish to achieve the desired properties in reduced space, which is a common requirement in modern applications.
\end{abstract}

Keywords: Essentially nonlinear negative stiffness; Equilibrium; Stability. 


\section{Introduction}

Negative stiffness elements have been utilized by researchers in an effort to mitigate vibrations in engineering structures. They involve a reversal of the usual directional relationship between force and displacement by assisting the imposed deflection. For example, buckled tubes exhibit negative stiffness to perturbations about a deformed state, where snap-through could also occur. Platus [1] and Trimboli et al. [2] have used springs with negative and positive stiffness coefficients in parallel configurations to achieve vibration isolation in a suspension system. A new design to achieve vibration isolation was proposed in [3] by setting up a serial connection between two springs of negative and positive stiffness coefficients (active vibration isolation). This configuration can lead to infinite stiffness against external disturbances. Tests were conducted on a bi-stable element (pre-buckled beam) that has been shown theoretically to exhibit negative stiffness behaviour [4]. The results indicated that a negative stiffness system has the ability to increase damping. An approach - based on the theory of thin shells - to design springs in order to minimize the suspension stiffness, and thus the fundamental frequencies for vehicle vibration isolation, was presented in [5]. There, a generic model of a spring element with variable negative stiffness was proposed. The mechanism was used in seat suspensions and was tested in vehicles, construction equipment and agricultural machines [6]. Similarly, a highly deformed slender beam attached to a vertically oscillating base was used in a vibration isolation application [7]. The extent of the beam's deflection was used to tailor its stiffness to achieve desirable isolator characteristics. The numerical results were verified by experimental measurements.

The stability of systems with negative stiffness elements has been analysed in [8]. A model of spring networks was used, and was found to be stable at the equilibrium point when tuned for high compliance but unstable when tuned for high stiffness. Additionally, it was found that a negative stiffness element, though unstable on its own, can be stabilized by including it in a system of positive stiffness elements [8]. 
Wang [9] has provided a summary of developments in this field, focused on the stability of negative stiffness discrete mechanical systems. Additionally, the equivalence among negative stiffness, pre-load and geometric nonlinearity was demonstrated by using discrete mechanical systems [9]. The static characteristics of a quasi-zero stiffness mechanism were investigated in [10]. A negative stiffness element was used to achieve low stiffness without large static deflection values in a three-spring configuration. Optimization between the geometry and the relative stiffness values of the springs was conducted to achieve a large excursion from the static equilibrium position, such that the stiffness of the system does not exceed a prescribed value. An approximate polynomial expression for the stiffness of the system was also derived. A non-linear bi-stable system with negative stiffness was investigated in [11] in terms of its energy harvesting capabilities. Static analysis revealed how the spring arrangement can produce a region with negative stiffness. It was shown that the amount of energy harvested is at most $4 / \pi$ times greater than that of the tuned linear absorber when the snap-through mechanism produces a square-wave-like response.

In this work, a new methodology is proposed to tailor strongly nonlinear negative stiffness for mechanical applications. This is primarily based on varying the path of motion of the free end of a preloaded linear spring element, as well as its geometrical properties. Three different paths of motion are examined and preliminary results of the force and stiffness variation characteristics are presented.

\section{Methodology}

The system for achieving nonlinear negative stiffness is shown in Figure 1. The linear spring of constant $k_{s}$ is unloaded at position OP, where its length is $l_{0}$. At any other position the length of the compressed (or extended) spring is determined by the coordinates $(x, y)$ of the path, along which the free end is prescribed to move. The end $\mathrm{O}$ of the spring is fixed. The coordinate system $(x, y)$ has its origin at point $A$. At this particular orientation the length of the spring is equal to $\mathrm{I}$. 
The force component acting in the y direction while the free end of the spring is moving on a prescribed path at a random location $\mathrm{C}$, is

$$
\begin{aligned}
& F(x, y)=(O C-O P) k_{S} \sin \alpha= \\
= & \left(\frac{O B}{\cos \alpha}-O P\right) k_{S} \sin \alpha= \\
= & \left(\frac{l+x}{\cos \alpha}-l_{0}\right) k_{S} \sin \alpha= \\
= & k_{S} y\left(1-\frac{l_{0}}{\sqrt{y^{2}+(l+x)^{2}}}\right)
\end{aligned}
$$

where the angle $\alpha$ is given by

$$
\tan \alpha=\frac{B C}{O B}=\frac{B C}{O A+A B}=\frac{y}{l+x}
$$

The path of the curve that the free end of the spring follows can take any shape according to the design requirements. The following three curves (plotted in Figure 2) are proposed to examine the effect on the variation of the nonlinear force produced,

$$
\begin{aligned}
& y=c_{1} x \\
& y=c_{2} x^{2} \\
& y=c_{3} x^{3}
\end{aligned}
$$

where $c_{1}, c_{2}$ and $c_{3}$ are constants. As it can be seen by comparing Figures 1 and 2 , the reference position A has been conveniently chosen to correspond to the zero-slope meeting point of the quadratic and cubic path curves. Combinations of the above equations can be introduced, in order to build more complicated profile shapes as per the specific application considered. 
After dividing both parts of equation (1) by $k_{s} l_{0}$

$$
\frac{F(x, y)}{k_{s} l_{0}}=y\left(\frac{1}{l_{0}}-\frac{1}{\sqrt{y^{2}+(l+x)^{2}}}\right) .
$$

and introducing the following transformations $[10,11]$,

$$
\hat{F}(x, y)=\frac{F(x, y)}{k_{s} l_{0}}, \hat{x}=\frac{x}{l_{0}}, \hat{y}=\frac{y}{l_{0}} \text { and } \gamma=\frac{l}{l_{0}},
$$

equation (3) takes the form

$$
\begin{gathered}
\hat{F}(\hat{x}, \hat{y})=\hat{y}\left\{1-\left[\hat{y}^{2}+(\gamma+\hat{x})^{2}\right]^{-1 / 2}\right\} . \\
y=c_{3} x^{3} .
\end{gathered}
$$

The parameter $\gamma$ is a geometrical parameter. When $\gamma=0$, the spring is initially vertical and when $\gamma=1$, the free end of the spring is crossing the origin of the coordinate system. By varying the parameter $\gamma$, a series of graphs of force-deflection characteristics $(\hat{F}-\hat{y})$ is obtained for the three different paths of motion of the linear spring's free end, which were presented in Figure 2. The results are depicted in Figure 3. As it can be seen, all three paths of motion exhibit variations in the slope of the force, which changes from positive to negative and to positive again for $0 \leq \gamma \leq 1$. The size of the area of negative slope (stiffness) changes according to the path type of the free end of the linear spring and the value of the geometrical parameter $\gamma$. Clearly, the system exhibits strongly nonlinear characteristics.

The non-dimensional stiffness, $\widehat{K}=\frac{k}{k_{s}}$, of the system can be calculated by differentiating the force equation (4) with respect to the displacement to give the following expressions:

$$
\begin{aligned}
& \widehat{K}=1-\frac{1}{\sqrt{\hat{y}^{2}+(\hat{x}+\gamma)^{2}}}+\frac{\hat{y}^{2}+\hat{x} \gamma+\hat{x}^{2}}{\left[\hat{y}^{2}+(\gamma+\hat{x})^{2}\right]^{3 / 2}}, \text { when } y=c_{1} x \\
& \widehat{K}=1-\frac{1}{\sqrt{\hat{y}^{2}+(\hat{x}+\gamma)^{2}}}+\frac{\hat{y}^{2}+0.5 \hat{x} \gamma+0.5 \hat{x}^{2}}{\left[\hat{y}^{2}+(\gamma+\hat{x})^{2}\right]^{3 / 2}}, \text { when } y=c_{2} x^{2}
\end{aligned}
$$




$$
\widehat{K}=1-\frac{1}{\sqrt{\hat{y}^{2}+(\hat{x}+\gamma)^{2}}}+\frac{\hat{y}^{2}+\frac{1}{3} \hat{x} \gamma+\frac{1}{3} \hat{x}^{2}}{\left[\hat{y}^{2}+(\gamma+\hat{x})^{2}\right]^{3 / 2}}, \text { when } y=c_{3} x^{3}
$$

A series of stiffness-deflection plots $(\widehat{K}-\hat{y})$ is obtained for the three different paths of motion by varying the parameter $\gamma$ (corresponding to the cases presented in Figure 3). The results are shown in Figure 4, where the dotted line corresponds to zero stiffness value, so that the transition points (from positive to negative stiffness and vice versa) are easily distinguishable. In Figures 4(b) and (c), the graphs that correspond to $\gamma$ values of $0,0.1$ and 0.2 have been confined to the axis ordinate limit values. A general trend observed is that the higher are the values of parameter $\gamma$ (smaller angles of the initial orientation of the spring) the larger is the size of the negative stiffness region, particularly in the cases of quadratic and cubic paths of motion. In addition, for lower values of $v$ (larger angles of the initial orientation of the spring), the minimum values of negative stiffness are much lower. On the other hand, the curves corresponding to $\gamma=1$ appear to be weakly nonlinear, while the negative stiffness region begins for deflection values $\hat{y}<0$. The region of the negative stiffness is also wider when $\hat{y}<0$ compared to the other $\gamma$ curves.

A bi-stable configuration that exhibits negative stiffness characteristics, comprising two oblique springs, has been presented in [10]. In this case, the path of motion is vertical and the stiffness reaches its minimum at the static equilibrium position. The stiffness variation of the configuration of reference [10] has been compared to that of the examined system for the cases when $\gamma=0.1$ and 0.4 (for all three different paths of motion). The results are presented in Figure 5. As it can be seen, the oblique springs achieve a broader area of negative stiffness compared to the case of a spring with varying path of motion. However, the minimum stiffness values obtained are lower in the latter case (quadratic and cubic paths), while the symmetry around the origin of the coordinate system is now broken. 
It is interesting to examine what would be the variation in the force and stiffness characteristics of the system when the equation of the path of motion is modified by altering the value of the participating constant. Three different force-deflection and stiffness curves are presented in Figure 6, corresponding to paths with equation $y=c_{3} x^{3}$ for three different values of $c_{3}$ and fixed value of $y=0.4$. It can be seen that as the constant value decreases, there is a decrease in the size of the region where negative stiffness is achieved. Additionally the minimum value of negative stiffness decreases as the constant value decreases (the corresponding values lay outside the boundaries of the graphs).

From the graphs of Figure 3 it can be observed that the positions where force becomes zero are altered, thus affecting the equilibrium positions of the system. The latter locations $\hat{y}_{1,2,3}$ are calculated by finding the roots of equation (4), which correspond to the positions where the vertical force is zero. This provides the following relations:

$$
\begin{aligned}
& \hat{y}_{1}=0 \\
& \hat{y}_{2}=\sqrt{1-(\gamma+\hat{x})^{2}} \\
& \hat{y}_{3}=-\sqrt{1-(\gamma+\hat{x})^{2}} .
\end{aligned}
$$

It can be concluded that equilibrium positions (9) and (10) can be modified according to the equation that describes the path of motion of the free spring end and the geometrical parameter $\gamma$. For simplicity reasons and in order to keep the presented expressions as less involved as feasible, the analysis below is confined to the case of the linear path, $y=c_{1} x$. Expression for cases of quadratic and cubic paths of motion can be derived in a similar manner. Equations (9) and (10) are equivalent to the following expressions (after substituting the linear motion path equation that relates coordinates $\mathrm{x}$ and $\mathrm{y}$ ):

$$
\hat{y}_{2}=\frac{-c_{1} \gamma-c_{1} \sqrt{1+c_{1}^{2}\left(1-\gamma^{2}\right)}}{1+c_{1}^{2}}
$$




$$
\hat{y}_{3}=\frac{-c_{1} \gamma+c_{1} \sqrt{1+c_{1}^{2}\left(1-\gamma^{2}\right)}}{1+c_{1}^{2}}
$$

Figure 7 exhibits the variation of the above equilibrium space with respect to parameters $\gamma$ and $c_{1}$. It can be seen that when small $c_{1}$ values are chosen, both equilibrium points lay in the vicinity of the origin point $A$ in the spring path (see Figure 1). Similar observation can be made when $\gamma$ approaches unity.

It is of interest to examine the stability properties of these equilibrium positions. This can be achieved by calculating the potential energy of the system, $P$. Then, the sign of the quantity $\frac{\partial^{2} P}{\partial \hat{y}^{2}}$ is determined. When $\frac{\partial^{2} P}{\partial \hat{y}^{2}}>0$, the equilibrium is stable, while for $\frac{\partial^{2} P}{\partial \hat{y}^{2}}<0$, the equilibrium is unstable. When $\frac{\partial^{2} P}{\partial \hat{y}^{2}}$ cannot be defined or is equal to zero, the system is marginally stable. It is found that for $y=c_{1} x$, equilibrium positions (9) and (10) are stable, while equilibrium position (8) is unstable. Additionally, the symmetry of the equilibrium positions (9) and (10) with respect to $\hat{y}=0$ is now broken. Figure 8 presents $\frac{\partial^{2} P}{\partial \hat{y}^{2}}-\hat{y}$ plots of the system when $y=c_{1} x$.

The corresponding values of equilibrium locations are:

- For $\gamma=0.4, \hat{y}_{1}=0, \hat{y}_{2}=0.478233$ and $\hat{y}_{3}=-0.878233$

- For $\gamma=0.6, \hat{y}_{1}=0, \hat{y}_{2}=0.34$ and $\hat{y}_{3}=-0.94$

When $y=c_{2} x^{2}$, equation (10) gives an imaginary number (marginally stable), while equilibrium position (9) is stable and equilibrium position (8) is unstable. Similar results occur when $y=c_{3} x^{3}$. Therefore, the choice of the path that the free end of the spring follows has a profound effect on the stability properties around the equilibrium positions of the system.

The relationship between force and deflection - as graphically shown in Figure 3 - is nonlinear. It would be useful to expand it in a Taylor series around the aforementioned equilibrium positions in order to 
highlight the main participating polynomial terms and the weight they carry. Therefore, when $\hat{y}=0$, the expansion of equation (4) in a Taylor series gives the following expression:

$$
F(\hat{x}, \hat{y}) \approx\left(1-\frac{1}{\sqrt{(\gamma+\hat{x})^{2}}}\right) \hat{y}+\frac{\sqrt{(\gamma+\hat{x})^{2}} \hat{y}^{3}}{2(\gamma+\hat{x})^{4}}
$$

In the case of linear path, equation (8) becomes (after substituting the relation between $\hat{x}, \hat{y}$ ):

$$
F(\hat{y}) \approx\left(1-\frac{1}{\gamma}\right) \hat{y}+\frac{\gamma \hat{y}^{2}}{c_{1} \gamma^{3}}+\frac{\left(0.5 c_{1}^{2}-1\right) \hat{y}^{3}}{c_{1}^{2} \gamma^{3}}
$$

The linear part of the above force equation can be eliminated when $\gamma=1$ (the free end of the spring is initially at the origin A). An approximate expression for the stiffness can be derived by differentiating equation (12) to give:

$$
\widehat{K}(\hat{y}) \approx\left(1-\frac{1}{\gamma}\right)+\frac{2 \gamma \hat{y}}{c_{1} \gamma^{3}}+\frac{\left(1.5 c_{1}^{2}-3\right) \hat{y}^{2}}{c_{1}^{2} \gamma^{3}}
$$

When $\gamma=1$, the constant term in the above equation is eliminated. The effect that parameters $c_{1}$ and $\gamma$ have on the stiffness is presented in Figure 9, where stiffness curves have been produced for various $c_{1}$ and $\gamma$ values. In Figure 9(a), $c_{1}=1$ for all curves while in Figure 9(b), $\gamma=0.4$.

It can be seen that stiffness is negative in the vicinity of $\hat{y}=0$ for a vast range of parameter $\gamma$ values (the curves of Figure 9(a) have been confined within the plot limits for visualisation purposes). For the case where $y=1$, negative stiffness is achieved only when $\hat{y} \leq 0$. In addition, lower $c_{1}$ values lead to greater negative stiffness magnitudes, as Figure 9(b) shows. Analytical expressions can be derived to describe the relation between parameters $c_{1}$ and $\gamma$ that lead to zero stiffness. However, the complexity of these functions prohibits their presentation in this article.

On the other hand, when using equation $\left(9^{\prime}\right)$, the expansion of the force - deflection function gives 


$$
\begin{gathered}
F(\hat{x}, \hat{z}) \approx-(-1+\gamma+\hat{x})(1+\gamma+\hat{x}) \hat{z}+\frac{3}{2}(\gamma+\hat{x})^{2} \sqrt{1-(\gamma+\hat{x})^{2}} \hat{z}^{2} \\
+\frac{1}{2}(\gamma+\hat{x})^{2}\left(-4+5(\gamma+\hat{x})^{2}\right) \hat{z}^{3}
\end{gathered}
$$

where $\hat{z}=\hat{y}-\sqrt{1-(\gamma+\hat{x})^{2}}$ [10]. By comparing equations (11) and (14), it can be seen that the nonlinear force expressions around the equilibrium positions can be tailored accordingly by choosing the equation that describes the path of motion. Additionally, the complexity of the forcing function is affected, since this differs when switching between the aforementioned equilibrium positions. In the case of equilibrium location $\left(9^{\prime}\right)$, equation (14) gives:

$$
\begin{gathered}
F(\widehat{w}) \approx\left[\frac{1+c_{1}^{2}\left(1-\gamma^{2}\right)+\gamma \sqrt{1+c_{1}^{2}\left(1-\gamma^{2}\right)}}{1+c_{1}^{2}}\right] \widehat{w}+ \\
\gamma\left[\frac{1+c_{1}^{2}\left(1-1.5 \gamma^{2}-1.5 \gamma \sqrt{1+c_{1}^{2}\left(1-\gamma^{2}\right)}\right)}{c_{1}\left(1+c_{1}^{2}\right)}\right] \widehat{w}^{2}+ \\
\gamma\left[\frac{\sqrt{1+c_{1}^{2}\left(1-\gamma^{2}\right)}}{c_{1}^{2}}+\frac{\gamma\left(-2-2 c_{1}^{2}+2.5 c_{1}^{2} \gamma^{2}-2.5 \gamma \sqrt{1+c_{1}^{2}\left(1-\gamma^{2}\right)}\right)}{1+c_{1}^{2}}\right] \widehat{w}^{3}
\end{gathered}
$$

where $\widehat{w}=\hat{y}-\frac{-c_{1} \gamma-c_{1} \sqrt{1+c_{1}^{2}\left(1-\gamma^{2}\right)}}{1+c_{1}^{2}}$. Again, it is found that the linear part of the above force equation can be eliminated when the following conditions are met (we note that only the positive roots are accepted):

$$
\gamma= \pm 1 \text { and } \gamma= \pm \frac{\sqrt{1+c_{1}^{2}}}{c_{1}}
$$

As $c_{1}$ increases, $\gamma$ asymptotically approaches unity. An approximate expression for the stiffness can be derived by differentiating equation (15) to give: 


$$
\begin{gathered}
\widehat{K}(\widehat{w}) \approx\left[\frac{1+c_{1}^{2}\left(1-\gamma^{2}\right)+\gamma \sqrt{1+c_{1}^{2}\left(1-\gamma^{2}\right)}}{1+c_{1}^{2}}\right]+ \\
2 \gamma\left[\frac{1+c_{1}^{2}\left(1-1.5 \gamma^{2}-1.5 \gamma \sqrt{1+c_{1}^{2}\left(1-\gamma^{2}\right)}\right)}{c_{1}\left(1+c_{1}^{2}\right)}\right] \widehat{w}+ \\
3 \gamma\left[\frac{\sqrt{1+c_{1}^{2}\left(1-\gamma^{2}\right)}}{c_{1}^{2}}+\frac{\gamma\left(-2-2 c_{1}^{2}+2.5 c_{1}^{2} \gamma^{2}-2.5 \gamma \sqrt{1+c_{1}^{2}\left(1-\gamma^{2}\right)}\right)}{1+c_{1}^{2}}\right] \widehat{w}^{2}
\end{gathered}
$$

The condition (16) eliminates the stiffness constant term. It was found that stiffness takes only positive values in the vicinity of this equilibrium position; therefore, there is no useful range of deflection for the achievement of negative stiffness.

Finally, in the case of equilibrium location (10'), equation (14) gives:

$$
\begin{gathered}
F(\widehat{w}) \approx\left[\frac{1+c_{1}^{2}\left(1-\gamma^{2}\right)-\gamma \sqrt{1+c_{1}^{2}\left(1-\gamma^{2}\right)}}{1+c_{1}^{2}}\right] \widehat{w}+ \\
\gamma\left[\frac{1+c_{1}^{2}\left(1-1.5 \gamma^{2}+1.5 \gamma \sqrt{1+c_{1}^{2}\left(1-\gamma^{2}\right)}\right)}{c_{1}\left(1+c_{1}^{2}\right)}\right] \widehat{w}^{2}+ \\
\left(-1-\frac{1 .}{c_{1}^{2}}+1.5 \gamma^{2}+\frac{2 \sqrt{1+c_{1}^{2}\left(1-\gamma^{2}\right)}\left(0.5+c_{1}^{2}\left(0.5-1.25 \gamma^{2}\right)\right)\left(-\gamma+\sqrt{1+c_{1}^{2}\left(1-\gamma^{2}\right)}\right)}{c_{1}^{2}\left(1 .+c_{1}^{2}\right)}\right) \widehat{w}^{3}
\end{gathered}
$$

where $\widehat{w}=\hat{y}-\frac{-c_{1} \gamma+c_{1} \sqrt{1+c_{1}^{2}\left(1-\gamma^{2}\right)}}{1+c_{1}^{2}}$. The conditions of equation (16) are valid in this case, as well as for the elimination of the linear part of the force. An approximate expression for the stiffness is derived by differentiating equation (18) to give:

$$
\widehat{K}(\widehat{w}) \approx\left[\frac{1+c_{1}^{2}\left(1-\gamma^{2}\right)-\gamma \sqrt{1+c_{1}^{2}\left(1-\gamma^{2}\right)}}{1+c_{1}^{2}}\right]+
$$




$$
\begin{gathered}
2 \gamma\left[\frac{1+c_{1}^{2}\left(1-1.5 \gamma^{2}+1.5 \gamma \sqrt{1+c_{1}^{2}\left(1-\gamma^{2}\right)}\right)}{c_{1}\left(1+c_{1}^{2}\right)}\right] \widehat{w}+ \\
3\left(-1-\frac{1 .}{c_{1}^{2}}+1.5 \gamma^{2}+2 \frac{\sqrt{1+c_{1}^{2}\left(1-\gamma^{2}\right)}\left(0.5+c_{1}^{2}\left(0.5-1.25 \gamma^{2}\right)\right)\left(-\gamma+\sqrt{1+c_{1}^{2}\left(1-\gamma^{2}\right)}\right)}{c_{1}^{2}\left(1 .+c_{1}^{2}\right)}\right) \widehat{w}^{2}
\end{gathered}
$$

The effect that parameters $c_{1}$ and $\gamma$ have on the stiffness around this equilibrium position is considered in Figure 10, where stiffness curves have been produced for various $c_{1}$ and $\gamma$ values. In Figure 10(a) it was assumed that $c_{1}=1$ for all curves, while in Figure $10(\mathrm{~b})$ it was assumed that $\gamma=0.4$. It is only for $\gamma=$ 0.4 that negative stiffness is achieved around this equilibrium position for the graphs of Figure 10(a). However, by decreasing the value of constant $c_{1}$, the useful range of deflection $\widehat{w}$ around which negative stiffness is achieved increases (Figure 10(b)). Finally, by comparing Figures 9 and 10, it can be concluded that the lowest negative stiffness values are met in the vicinity of the equilibrium position $\hat{y}=0$.

\section{Concluding Remarks}

A new methodology to tailor negative nonlinear stiffness has been presented in this paper, based on the path of motion of the free end of a linear spring. This includes the realization of springs with essential negative stiffness nonlinearity, i.e. negative stiffness with no linear part. Three different curves have been proposed for this purpose, gradually increasing the complexity of the design: linear, quadratic and cubic paths of motion. It has been found that the extent of the deflection region where negative stiffness is achieved varies according to the curve type/participating constants, as well as the initial state of spring length. Additionally, the stability properties and the force-deflection functions around the equilibrium positions of the system are also affected. Analytical expressions for the overall stiffness in the vicinity of those equilibria have been produced for the case of linear path of motion, leading to the identification of useful deflection regions in terms of achievement of negative stiffness. Future research 
plans should address the effect of the inaccuracies induced due to manufacturing errors when constructing the examined paths of motion. An attractive configuration to implement the methodology presented in this work is potentially a slider mechanism. However, the profound implication is the development of friction in the contact area between the moving spring end and the groove. The effect of the friction force opposing the spring force could potentially lead to quasi-zero stiffness characteristics and requires a comprehensive analysis. Finally, experimental demonstration of the numerical work presented will be explored to validate the methodology presented, as part of future research activities.

\section{Acknowledgements}

Dr F. Liu wishes to express his gratitude to the National Natural Science Foundation of P.R. China for supporting this research (Grant No.51305378). 


\section{References}

1. D.L. Platus, Negative-stiffness-mechanism vibration isolation systems, Proceedings of the SPIE-The International Society for Optical Engineering, 3786 (1999) 98-105.

2. M.S. Trimboli, R. Wimmel and E. Breitbach, A quasi-active approach to vibration isolation using magnetic springs, Proceedings of the SPIE-The International Society for Optical Engineering, 2193 (1994), 73-83.

3. T. Mizuno, T. Toumiya and M. Takasaki, Vibration isolation system using negative stiffness, JSME International Journal Series C Mechanical Systems, Machine Elements and Manufacturing, 46 (2003), 807-812.

4. L. Kashdan, C. Seepersad, M. Haberman and P.S. Wilson, Design, fabrication and evaluation of negative stiffness elements using SLS, Rapid Prototyping Journal, 18 (2012) 194 - 200.

5. C.M. Lee, V.N. Goverdovskiy and A.I. Temnikov, Design of springs with "negative" stiffness to improve vehicle driver vibration isolation, Journal of Sound and Vibration, 302 (2007), 865-874.

6. C.M. Lee and V.N. Goverdovskiy, Alternative vibration protecting systems for men-operators of transport machines: modern level and prospects, Journal of Sound and Vibration 249 (2002), 635647.

7. L.N. Virginia, S.T. Santillan and R.H. Plaut, Vibration isolation using extreme geometric nonlinearity, Journal of Sound and Vibration, 315 (2008), 721-731.

8. Y.C. Wang and R.S. Lakes, Extreme stiffness systems due to negative stiffness elements, American Journal of Physics, 72 (2004), 40-50.

9. http://www.stam.org.tw/files/newsletter/newsletterTopicReport/WangYC.pdf (Accessed 23 August 2012).

10. A. Carrella, M.J. Brennan and T.P. Waters, Static analysis of a passive vibration isolator with quasizero-stiffness characteristic, Journal of Sound and Vibration, 301 (2007), 678-689.

11. R. Ramlan, M.J. Brennan, B.R. Mace and I. Kovacic, Potential benefits of a non-linear stiffness in an energy harvesting device, Nonlinear Dynamics, 59 (2010), 545-558. 


\section{Figure Captions}

Figure 1: Schematic representation of the proposed design exhibiting negative stiffness.

Figure 2: Examined paths of motion for the free end of the linear spring: (a) $y=c_{1} x$, (b) $y=c_{2} x^{2}$ and (c) $y=c_{3} x^{3}$.

Figure 3: Force-deflection characteristics of the system for the examined paths of motion of the linear spring's free end: (a) $y=c_{1} x$, (b) $y=c_{2} x^{2}$ and (c) $y=c_{3} x^{3}$.

Figure 4: Non-dimensional stiffness of the examined system, corresponding to the cases of Figure 3:

(a) $y=c_{1} x$, (b) $y=c_{2} x^{2}$ and (c) $y=c_{3} x^{3}$.

Figure 5: Comparison of the non-dimensional stiffness characteristics of the examined system to those of two oblique springs [10] for (a) $\gamma=0.1$ and $(b) \gamma=0.4$.

Figure 6: (a) Force and (b) stiffness characteristics of the system when $y=c_{3} x^{3}\left(c_{3}=100,300\right.$ and 500).

Figure 7: Space of the equilibrium positions $\hat{y}_{2}$ and $\hat{y}_{3}$, when $y=c_{1} x$.

Figure 8: $\frac{\partial^{2} P}{\partial \hat{y}^{2}}-\hat{y}$ plots of the system when $y=c_{1} x\left(c_{1}=1\right)$ for $\gamma=0.4$ and $\gamma=0.6$.

Figure 9: Stiffness variation around equilibrium position $\hat{y}=0$ with respect to (a) $\gamma$ and (b) $c_{1}$.

Figure 10: Stiffness variation around equilibrium position $\hat{y}=-\sqrt{1-(\gamma+\hat{x})^{2}}$ with respect to (a) $\gamma$ and (b) $c_{1}$. 


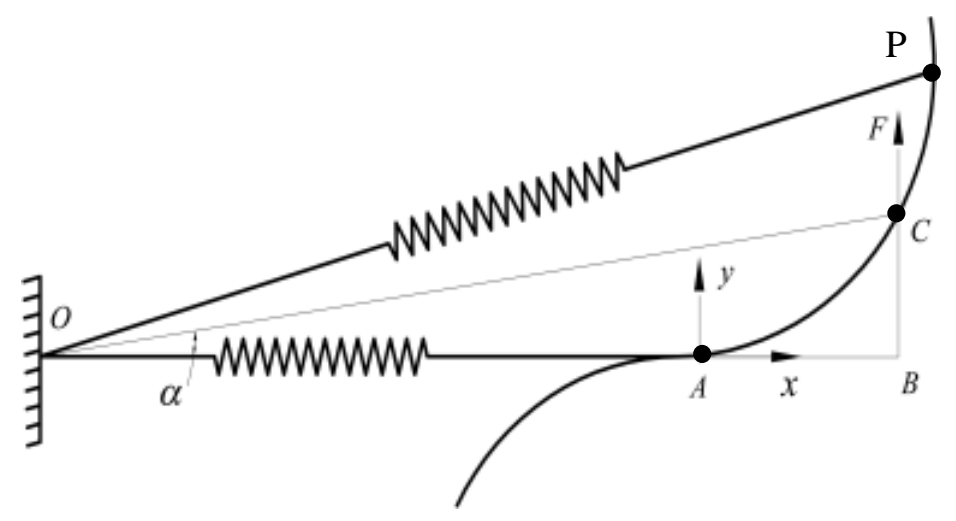

Fig1.tif 


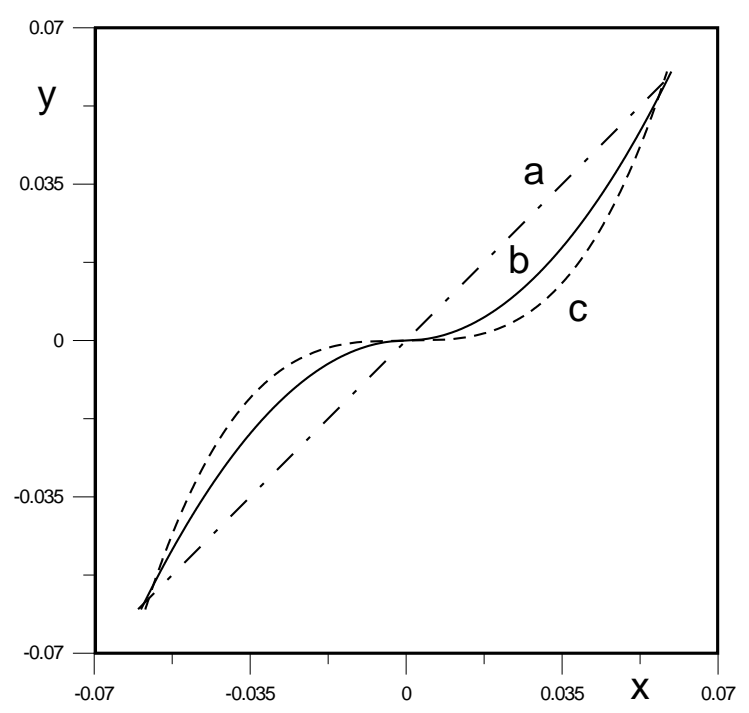

Fig2.tif 


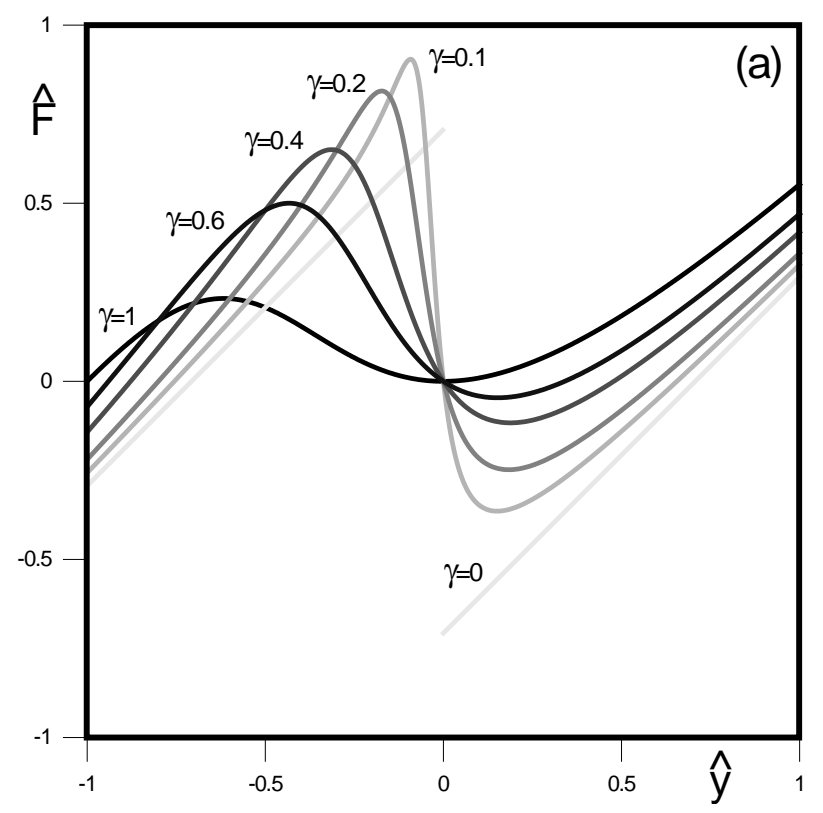

Fig3a.tif 


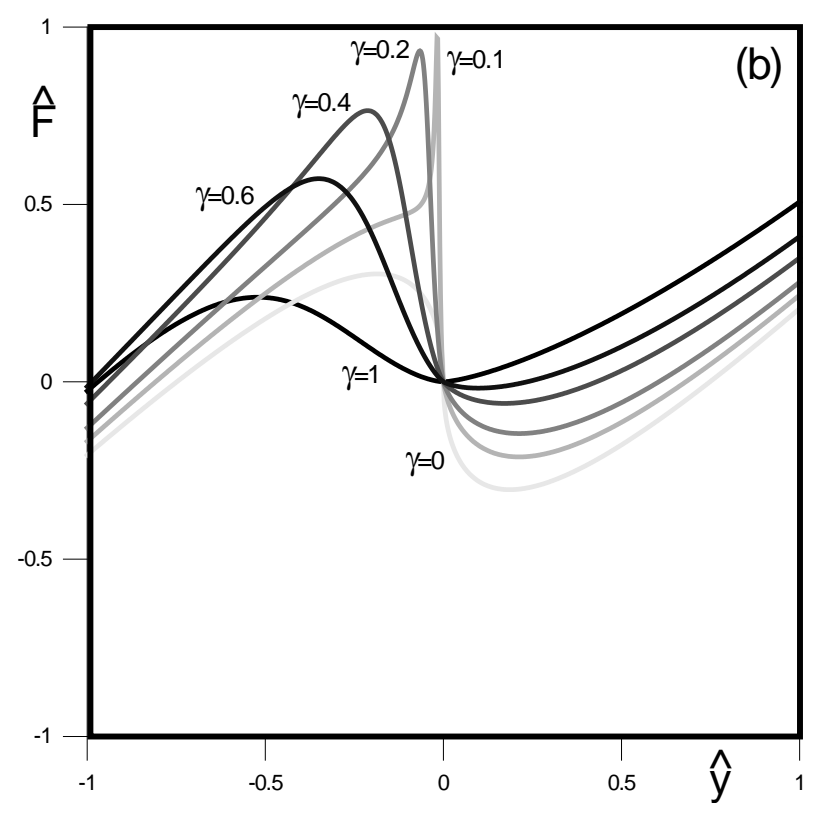

Fig3b.tif 


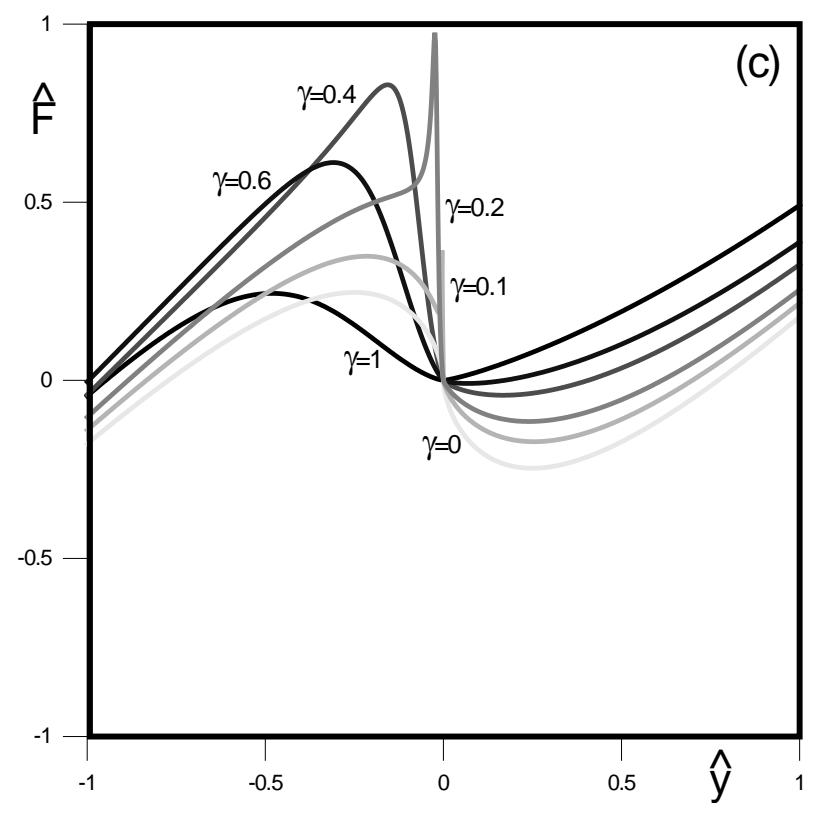

Fig3c.tif 


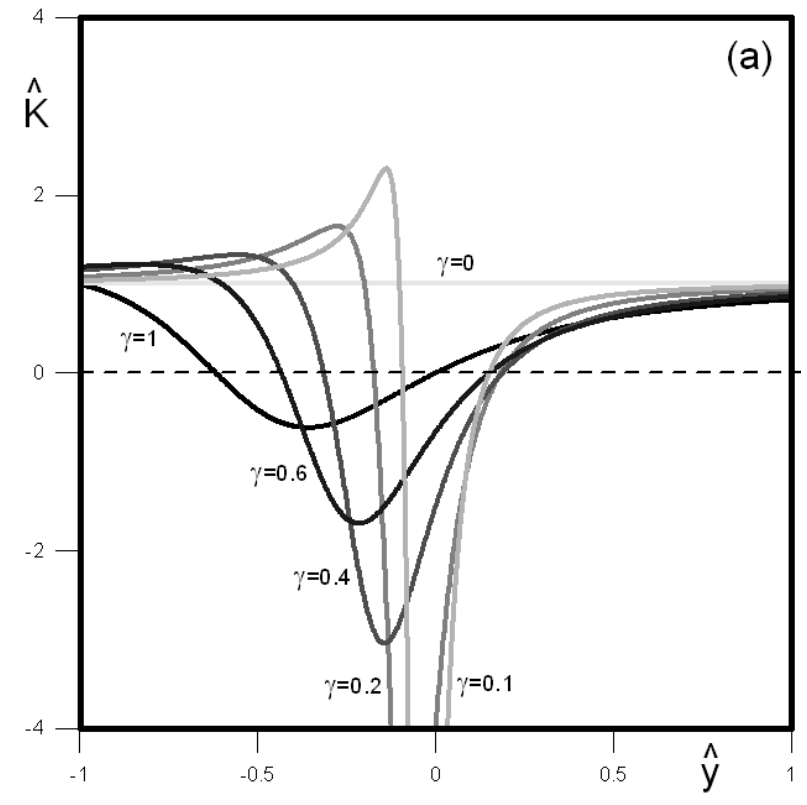

Fig4a.tif 


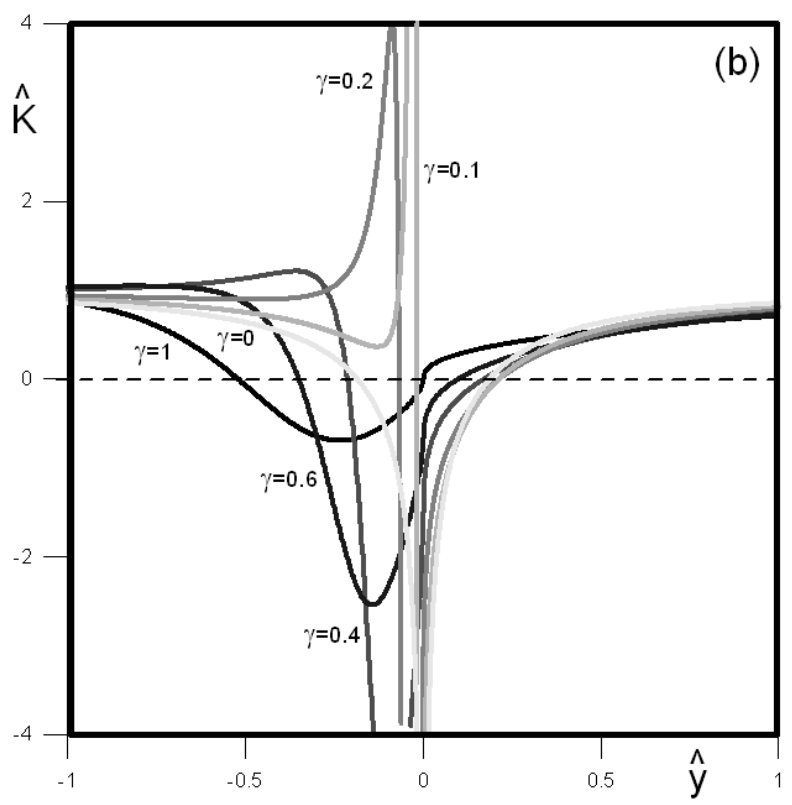

Fig4b.tif 


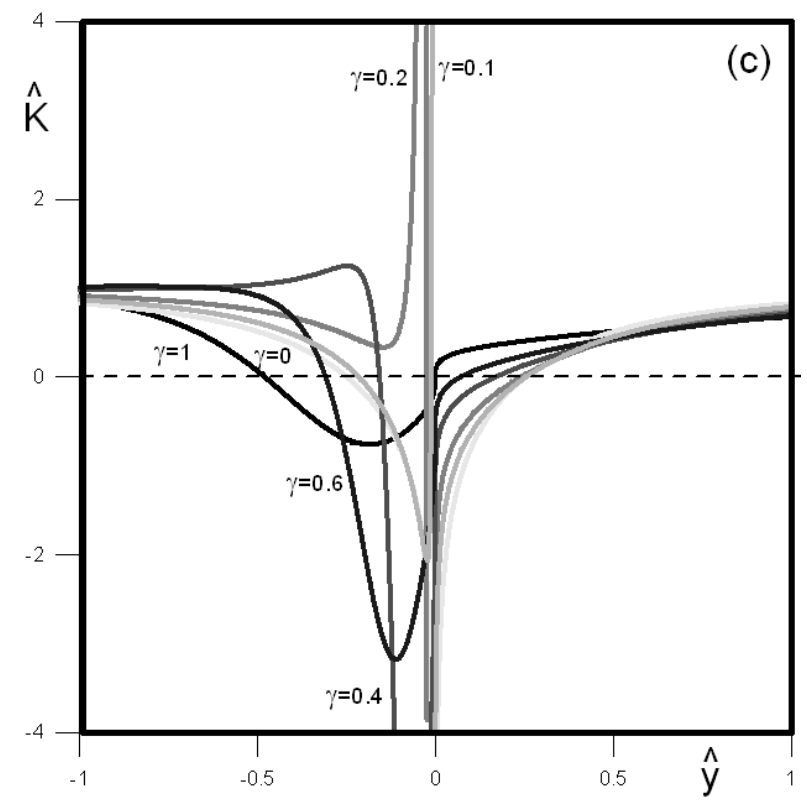

Fig4c.tif 


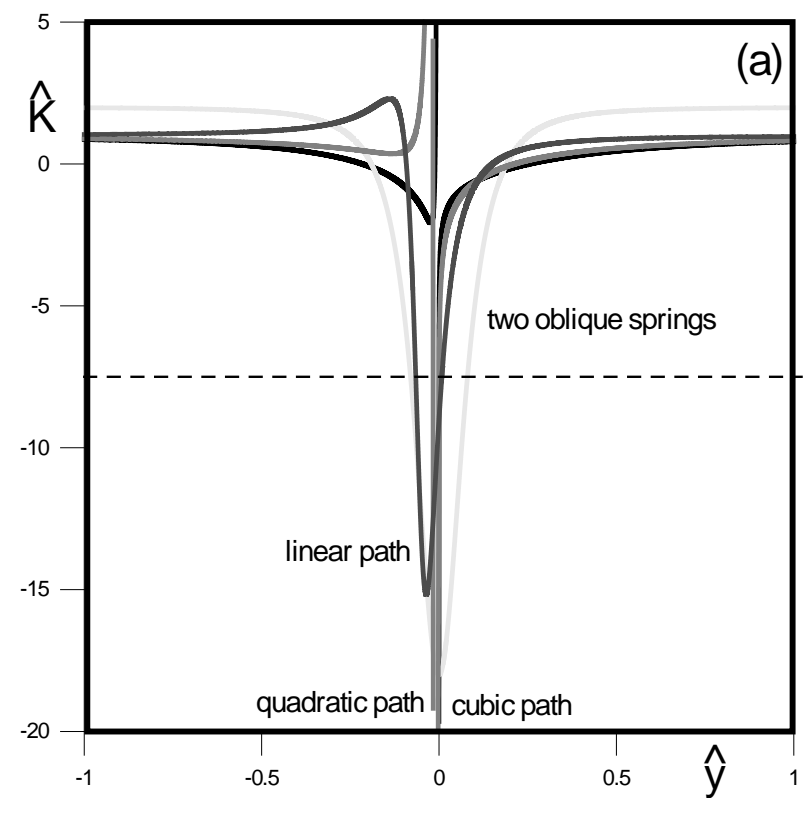

Fig5a.tif 


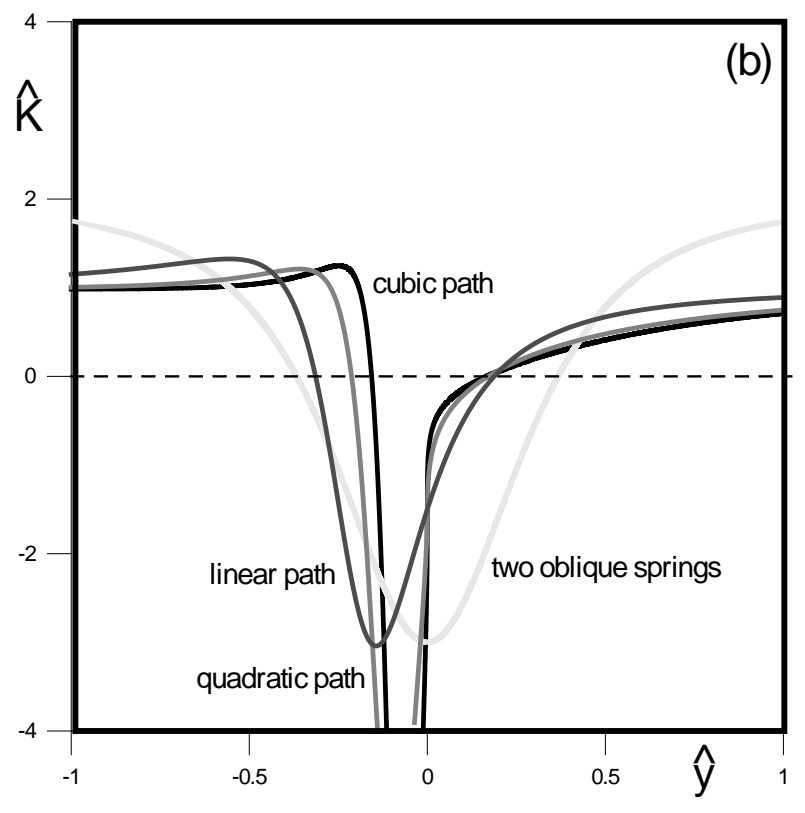

Fig5b.tif 


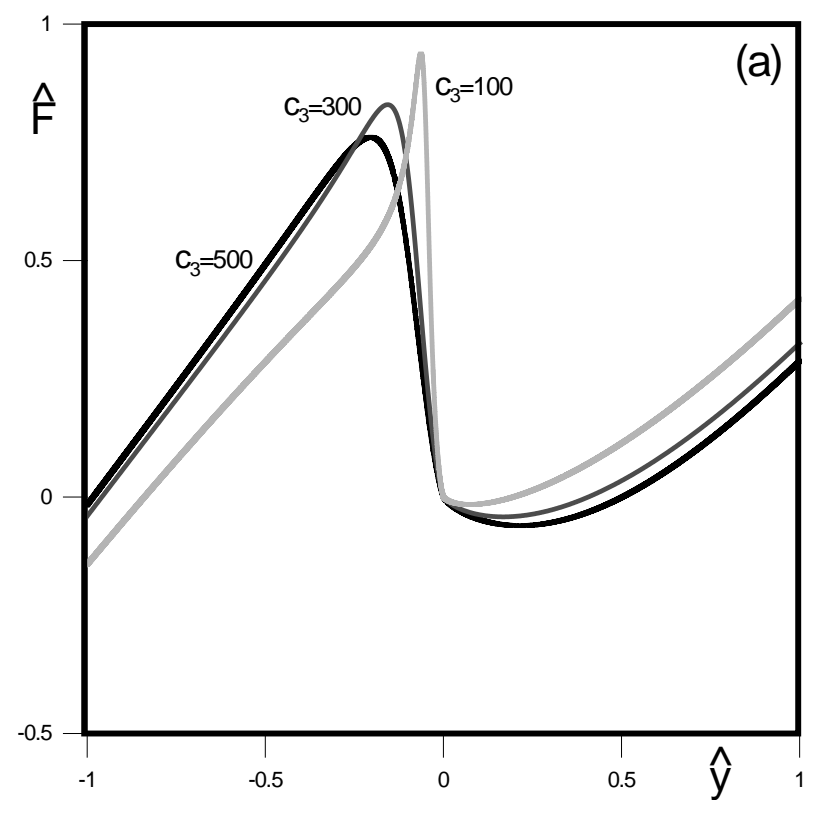

Fig6a.tif 


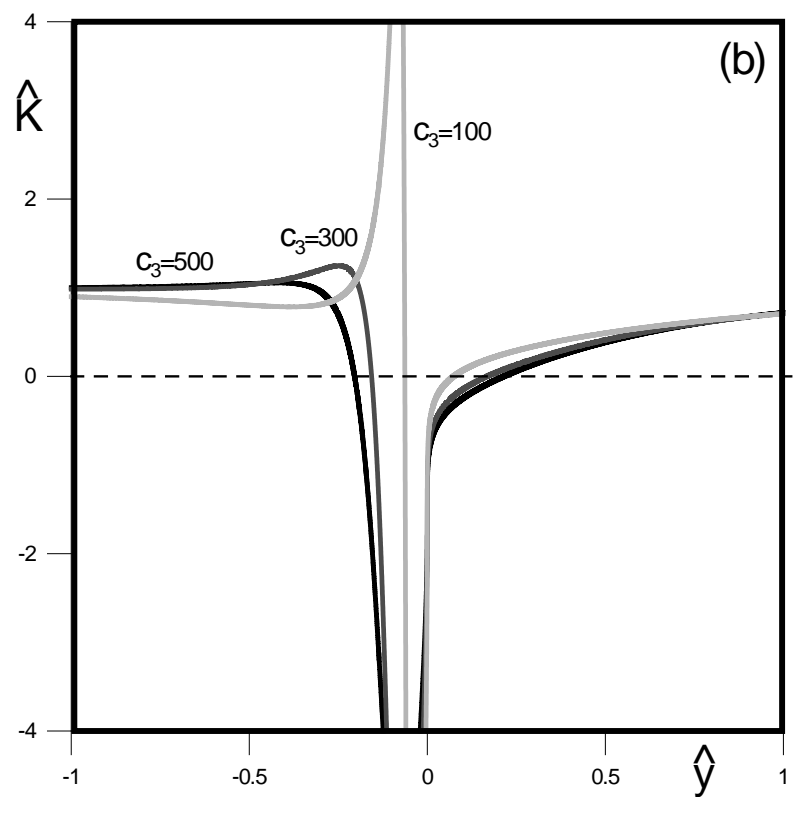

Fig6b.tif 


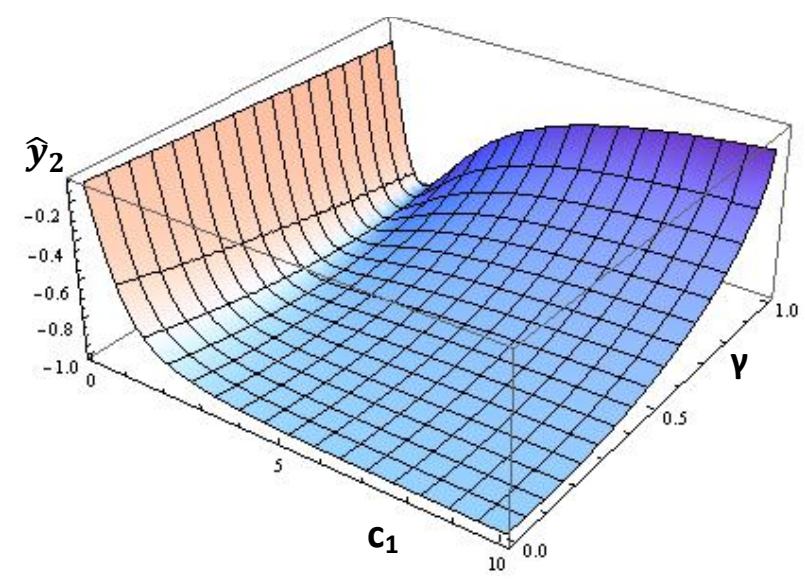

Fig7a.tif 


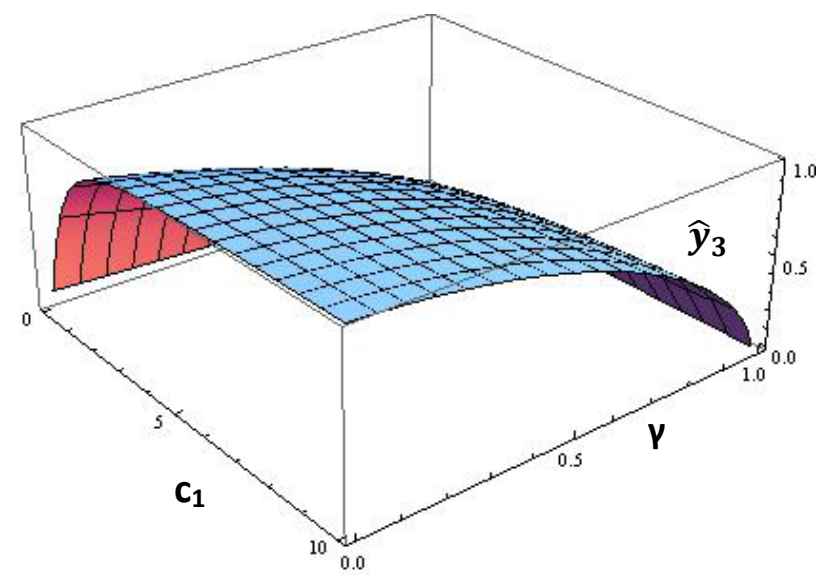

Fig7b.tif 


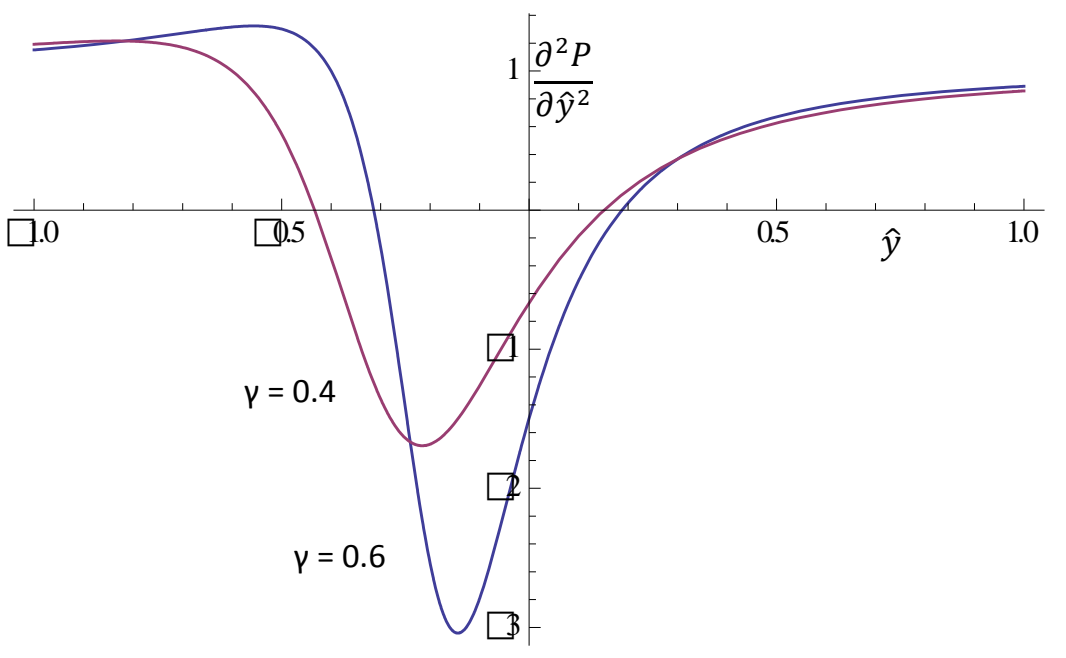

Fig8.tif 


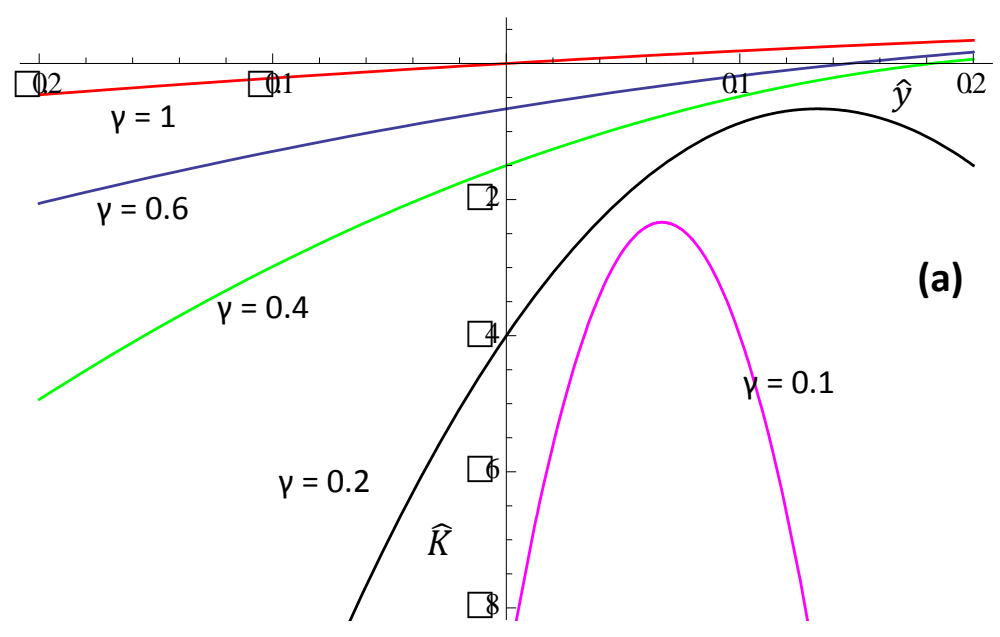

Fig9a.tif 


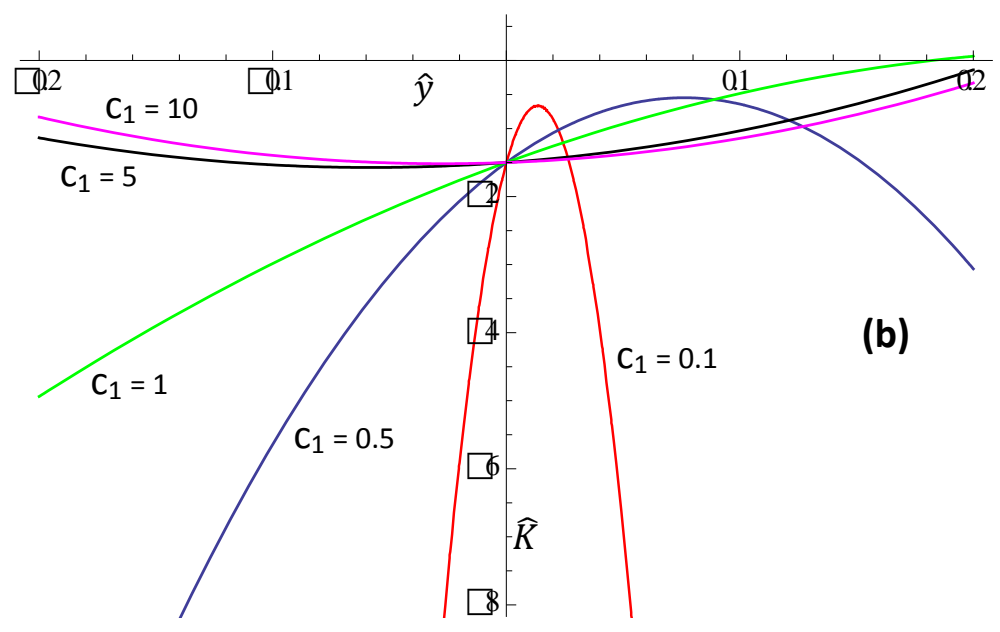

Fig9b.tif 


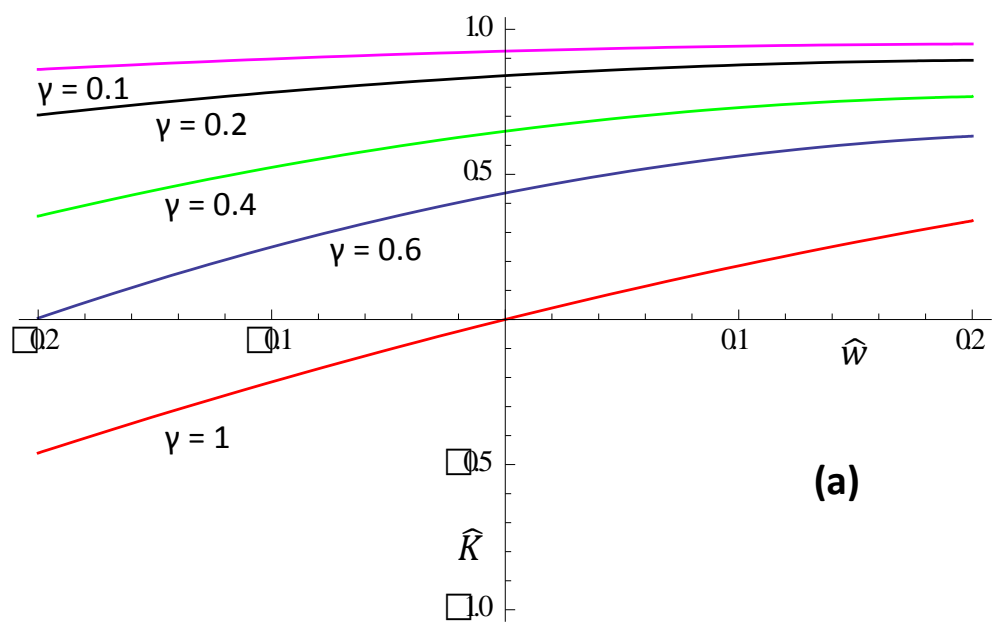

Fig10a.tif 


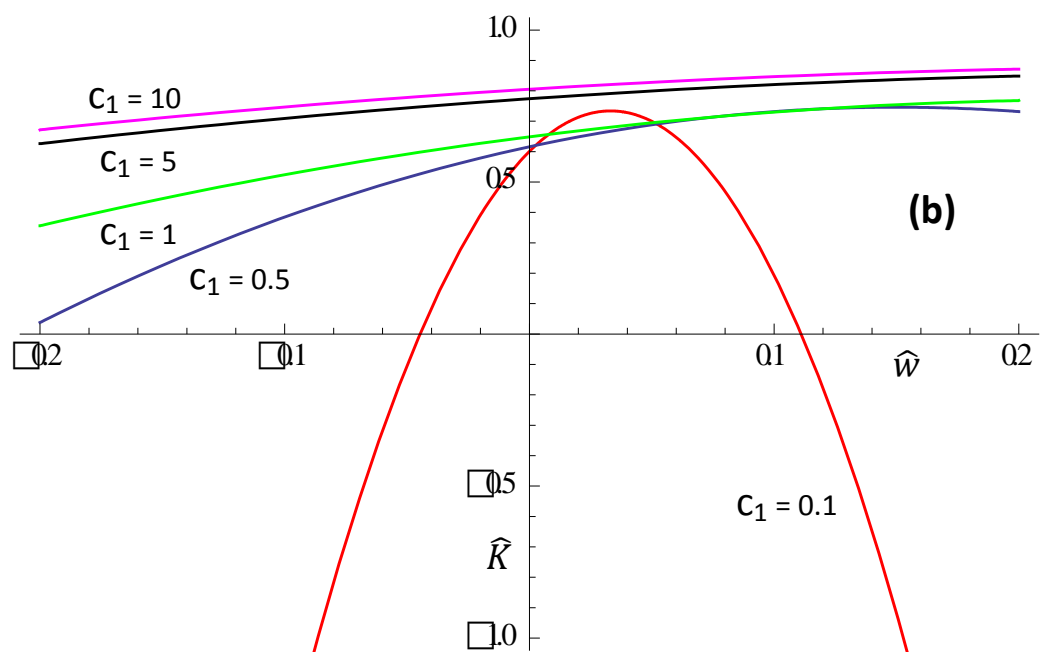

Fig10b.tif 